\title{
Increase in Adverse Reactions Associated with Use of Synthetic Cannabinoids - Anchorage, Alaska, 2015-2016
}

\begin{abstract}
Yuri P. Springer, $\mathrm{PhD}^{1,2}$; Roy Gerona, $\mathrm{PhD}^{3}$; Erich Scheunemann ${ }^{4}$; Sarah L. Shafer ${ }^{5}$; Thomas Lin 3 ; Samuel D. Banister, PhD 3,6 ; Michael P. Cooper, MD ${ }^{1}$; Louisa J. Castrodale, DVM ${ }^{1}$; Michael Levy, MD ${ }^{4}$; Jay C. Butler, $\mathrm{MD}^{7}$; Joseph B. McLaughlin, MD ${ }^{1}$
\end{abstract}

In July 2015, personnel in the Alaska Division of Public Health's Section of Epidemiology became aware of an increase in the number of patients being treated in Anchorage hospital emergency departments for adverse reactions associated with use of synthetic cannabinoids (SCs). SCs are a chemically diverse class of designer drugs that bind to the same cannabinoid receptors as tetrahydrocannabinol, the main psychoactive component of cannabis. A public health investigation was initiated to describe clinical outcomes, characterize the outbreak, and identify SC chemicals circulating in Anchorage. During July 15, 2015-March 15, 2016, a total of 1,351 ambulance transports to Anchorage emergency departments for adverse SC reactions were identified. A review of charts obtained from two Anchorage hospitals determined that among 167 emergency department visits for adverse SC reactions during July 15-September 30, 2015, 11 (6.6\%) involved a patient who required endotracheal intubation, 17 (10.2\%) involved a patient who was admitted to the intensive care unit, and $66(39.5 \%)$ involved a patient classified as being homeless. Testing of 25 product and paraphernalia samples collected from patients at one hospital identified 11 different SC chemicals. Educational outreach campaigns focused on the considerable health risks of using SCs need to complement judicial and law enforcement actions to reduce SC use.

The outbreak was recognized in July 2015, based on reports in local news media and after a report of a $330 \%$ increase in calls to U.S. Poison Control Centers regarding SC poisonings nationwide during January-April 2015 (1). Investigations of outbreaks of adverse reactions associated with use of SCs in other U.S. cities have documented health effects, including altered mental status, seizures, acute impairment of organ function, and death (2-6). Because of the potential for severe health outcomes associated with SC use, a public health investigation was initiated, focused on characterizing clinical outcomes and patient demographics, enumerating cases to estimate the associated burden on Anchorage emergency health care systems, and identifying SC chemicals circulating in Anchorage.

To characterize patient demographics and clinical outcomes, personnel in the Alaska Division of Public Health's Section of Epidemiology performed authorized electronic medical record (EMR) reviews and chart abstractions at two of the three nonmilitary hospitals in Anchorage with available records. The one military hospital in Anchorage was not included in the investigation. EMR data were searched using a standardized query that flagged emergency department visits associated with one or more of 11 discharge diagnoses* related to acute drug toxicity, altered mental status, and drug ingestion or abuse. An SC emergency department visit was defined as an emergency department visit during July 15-September 30, 2015, to either of the two designated hospitals by a patient with an adverse reaction attributed to self-reported or suspected SC use immediately preceding onset of the adverse reaction. Self-reported or suspected SC use was determined through review of charts of patients associated with flagged emergency department visits. Suspicion of use was based on clinical signs and symptoms deemed consistent with SC use by health care personnel caring for the patient and was often reinforced by the patient's possession of SCs or related paraphernalia or witness-reported observed SC use immediately before onset of adverse reactions.

Because EMR data were only available from two of three nonmilitary hospitals in Anchorage, ambulance transport data collected by the Anchorage Fire Department, which is responsible for all ambulance transports within Anchorage, were used to enumerate cases. An SC transport was defined as an ambulance transport during July 15, 2015-March 15, 2016 (the outbreak investigation period), to any of the three nonmilitary hospitals in Anchorage of a patient with an adverse reaction attributed to self-reported or suspected SC use immediately preceding onset of the adverse reaction. Evidence of self-reported or suspected SC use was obtained from ambulance transport data. Suspicion of use was based on clinical signs and symptoms deemed consistent with SC use by emergency medical services (EMS) personnel transporting the patient. On November 10, 2015, the Anchorage municipality assembly passed ordinance AO 2015-123(S) that raised the criminal penalties associated with possession, sale, and use of SCs in Anchorage. Potential effects of this ordinance on the outbreak were evaluated by comparing total ambulance transports, total and mean number of SC transports, and mean percentage of ambulance transports that were SC transports before and after the outbreak began and before and after passage of the ordinance.

\footnotetext{
${ }^{*}$ Keywords used in EMR search $=$ acute drug intoxication, altered mental status (including with qualifiers "unspecified" and "unspecified altered mental status"), drug abuse, drug ingestion, drug psychosis, drug use, illicit drug use, polysubstance abuse, and substance abuse.
} 
To identify SC chemicals circulating in Anchorage during the outbreak, emergency department personnel at one of the hospitals that participated in the EMR reviews (hospital A) collected 25 samples of products (e.g., plastic bags containing loose herbaceous material) and paraphernalia (e.g., pipes, storage containers, or plastic bags) from 14 patients treated for suspected SC use. These samples were analyzed by liquid chromatography quadrupole time-of-flight mass spectrometry at the University of California, San Francisco. In addition, biologic specimens (blood or urine) collected from three patients treated at hospital A for suspected SC use during the outbreak (one sample per patient) and postmortem serum specimens from 10 persons who died in Anchorage during the outbreak, were tested for secondary metabolites of SC chemicals.

Among 488 emergency department visits during July 15September 30, 2015 flagged by EMR query, a total of 167 (34.2\%) were classified as SC emergency department visits, including $74(44.3 \%)$ that involved self-reported SC use and $93(55.7 \%)$ that involved suspected SC use. These 167 SC emergency department visits involved 128 unique patients for whom full name and date of birth information was available; $104(81.3 \%)$ were male, and the median age was 34 years (range $=12-62$ years). Sixty-six (39.5\%) of these SC emergency department visits involved a patient classified as homeless, on the basis of self-report, address information provided to EMS personnel or emergency department staff members by the patient, or location of ambulance pickup. More than two thirds of these SC emergency department visits involved a patient who was lethargic in the emergency department, and one third involved a patient who was agitated. Eleven (6.6\%) SC emergency department visits involved a patient who required endotracheal intubation either en route to or while in the emergency department, and $17(10.2 \%)$ involved a patient who was admitted to an intensive care unit (Table 1).

Review of Anchorage Fire Department ambulance transport data collected during July 15, 2015-March 15, 2016 (the outbreak investigation period) identified 13,403 ambulance transports in Anchorage, among which 1,351 (10.1\%) were SC transports (Table 2). Most SC transports (956; 70.8\%) originated within a $2-\mathrm{km}^{2}$ area of downtown Anchorage that is known to be a hub for the local homeless population and associated service providers. The 1,351 SC transports involved 535 unique patients for whom full name and date of birth information was available; 426 (79.6\%) were male, and the median age was 34 years (range $=12-69$ years). Among the 535 patients, $199(37.2 \%)$ were transported more than once for adverse SC reactions during this period (mean $=4.6$ transports/patient; range $=2-24)$, and 17 patients $(3.2 \%$ of all patients) were transported 10 or more times, accounting for 267 (19.8\%) of all SC transports.
TABLE 1. Selected health outcomes and medical interventions associated with 167 Emergency Department (ED) visits for adverse reactions associated with use of synthetic cannabinoids - Anchorage, Alaska, July 15, 2015-September 30, 2015

\begin{tabular}{lr}
\hline Health outcome or medical intervention of interest & $\begin{array}{c}\text { ED visits } \\
\text { No. (\%) }\end{array}$ \\
\hline $\begin{array}{l}\text { Lethargy (confusion, somnolence, sleep, and } \\
\text { unresponsiveness) }\end{array}$ & $115(68.9)$ \\
Agitation (distress, anxiety, aggressiveness, and violence) & $55(32.9)$ \\
Both lethargy and agitation & $37(22.2)$ \\
Tachypnea (respiratory rate $>16$ breaths/min) & $76(45.5)$ \\
Tachycardia (heart rate $>100$ beats/min) & $52(31.1)$ \\
Required physical restraint en route to or while in ED & $26(15.6)$ \\
Endotracheal intubation for respiratory insufficiency or & $11(6.6)$ \\
airway protection en route to or while in ED & $17(10.2)$ \\
\hline Intensive care unit admission &
\end{tabular}

Before the investigation began, quantitative baseline data on rates of adverse SC reactions in Anchorage were not available. A retrospective review of Anchorage Fire Department ambulance transport data collected during January 1, 2014-July 14, 2015 (the preoutbreak period) identified 240 SC transports (mean $=0.4$ per day) $($ Table 2 ). During the portion of the outbreak investigation period before issuance of the ordinance (July 15-November 10, 2015), there were 844 SC transports (mean $=7.1$ per day) compared with 507 $\mathrm{SC}$ transports (mean $=4.0$ per day) after the ordinance was issued (November 11, 2015-March 15, 2016) (Table 2). The mean daily number of SC transports during the preordinance outbreak period (7.1) was 17.8 times higher than that during the preoutbreak period (0.4). Analysis of daily SC transport counts from the postordinance outbreak period ( $\mathrm{N}=126$ days) by linear regression identified a significant decline in counts over time $\left(\mathrm{r}^{2}{ }_{\text {adj }}=0.1 ; \mathrm{p}<0.001\right)$.

Eleven different $\mathrm{SC}$ chemicals ${ }^{\dagger}$ were identified among product and paraphernalia samples collected at hospital A, the most common being AB-CHMINACA, MAB-CHMINACA, and 5F-AMB, all highly potent cannabinoid receptor agonists $(7,8)$. Among the 17 (68.0\%) samples that tested positive for SCs, all contained multiple SC chemicals. Four of the SC-positive samples were clear, viscous liquids labeled as car air fresheners that can easily be inhaled by using a refillable electronic cigarette, and anecdotal reports from health care providers indicated that a subset of patients treated for adverse SC reactions admitted vaping SCs in liquid form. Among blood or urine specimens collected from three patients treated at hospital A for suspected SC use (one specimen per patient), one tested positive for a metabolite of $5 \mathrm{~F}-\mathrm{AMB}$ and another for a metabolite of MAB-CHMINACA. Postmortem serum specimens collected from 10 patients who died during the

\footnotetext{
$\dagger$ AB-CHMINACA, AB-FUBINACA, AB-PINACA, AKB48-N-5F-Pentyl analog, APP-CHMINACA, MAB-CHMINACA, NM2201, XLR-11, 5-Chloro-AB-PINACA, 5F-AB-PINACA, and 5F-AMB.
} 
TABLE 2. Ambulance transports, including those associated with adverse reactions associated with use of synthetic cannabinoids during preoutbreak period and outbreak period (before and after passage of ordinance AO 2015-123(S))* Anchorage, Alaska, January 1, 2014March 15, 2016

\begin{tabular}{|c|c|c|c|c|}
\hline \multirow[b]{3}{*}{ Epidemiologic parameter } & \multicolumn{4}{|c|}{ Investigation period } \\
\hline & \multirow[b]{2}{*}{ Preoutbreak period } & \multicolumn{3}{|c|}{ Outbreak period } \\
\hline & & Overall & Preordinance & Postordinance \\
\hline Dates & Jan 1, 2014-Jul 14, 2015 & Jul 15, 2015-Mar 15, 2016 & Jul 15, 2015-Nov 10, 2015 & Nov 11, 2015-Mar 15, 2016 \\
\hline No. days & 560 & 245 & 119 & 126 \\
\hline Total no. ambulance transports & 25,642 & 13,403 & 6,548 & 6,855 \\
\hline Total no. (\%) SC transports & $240(0.09)$ & $1,351(10.1)$ & $844(12.9)$ & $507(7.4)$ \\
\hline Daily mean no. ambulance transports & 45.8 & 54.7 & 55.0 & 54.4 \\
\hline Daily mean no. SC transports (range) & $0.4(0-4)$ & $5.5(0-25)$ & $7.1(0-25)$ & $4.0(0-11)$ \\
\hline $\begin{array}{l}\text { Daily mean percentage of all ambulance } \\
\text { transports that were SC transports (range) }\end{array}$ & $0.9(0-8.0)$ & $9.5(0-36.9)$ & $11.8(0-36.9)$ & $7.4(0-22.4)$ \\
\hline
\end{tabular}

Data source: Anchorage Fire Department.

Abbreviation: $\mathrm{SC}=$ synthetic cannabinoid.

* https://www.municode.com/library/api/CD/Ordinances/12717/747882?forceDownload=true.

outbreak were tested; four were positive for metabolites of SC chemicals, and for these decedents, SC use was noted as either a main or a contributing cause of death on the death certificate.

\section{Discussion}

An outbreak of adverse reactions associated with use of SCs occurred in Anchorage, involving at least 535 persons during at least 1,351 emergency department visits throughout the 245day outbreak investigation period (July 15, 2015-March 15, 2016). On average, daily SC transports increased nearly eighteenfold during the preoutbreak period and the period of the outbreak before passage of ordinance AO 2015-123(S), which raised criminal penalties associated with possession, use, and sale of SCs in Anchorage. Passage of the ordinance was associated with a significant decline in the number of SC transports.

Among all SC emergency department visits, 39.5\% involved homeless persons, although this number likely represents an underestimate, because homelessness was not consistently recorded in medical charts and might be difficult to ascertain by interview. No previous investigations into outbreaks of adverse reactions associated with use of SCs that documented a similar disproportionate impact on homeless persons could be found. The absence of concurrent outbreaks of adverse reactions in other Anchorage populations at increased risk for drug use, including high school students, suggests that local SC dealers might have targeted the homeless population.

The investigation identified 11 different SC chemicals circulating in Anchorage during the outbreak. All 17 SC-positive samples contained multiple SC chemicals, and some samples included more than one potent SC (7-9), suggesting the potential for interactive chemical effects that might intensify the severity of adverse reactions experienced by users. Several of the detected SC chemicals have been found to potently induce a response in an associated binding receptor (CB1) that is $132 \%-152 \%$ higher than that elicited by the full agonist molecule CP 55,940, widely used in pharmacological studies of the endocannabinoid system $(7,9)$. In addition, documentation of SCs in liquid form provides evidence for inhalation by means other than smoking of herbal materials, a finding not reported from investigations of similar outbreaks in other states.

The findings in this report are subject to at least two limitations. First, because not all nonmilitary hospitals participated in the initial chart review, findings related to clinical outcomes might not be representative. Second, because biologic specimens (blood or urine) were not systematically collected from all patients and tested, linkage of observed adverse reactions with use of SCs could only be inferred from patient history. Despite these limitations, this investigation revealed considerable adverse effects associated with SC use on the public's health and health care resources in Anchorage and underscores the need for continued measures to deter SC use nationally.

The primary interventions employed to control this outbreak were judicial and law enforcement actions to limit drug availability (i.e., passage of ordinance AO 2015-123(S) and increased efforts by police to identify and arrest local SC manufacturers and distributors) and educational outreach actions to reduce demand for the drug. During the outbreak, the Alaska Division of Public Health conducted educational outreach to stakeholders concerning the considerable health risks of SC use by posting fact sheets and other information on websites and social media platforms; participating in print, radio, and television media interviews; meeting directly with providers of services for the homeless population; participating in multiagency meetings with other critical partners including police and fire department personnel, behavioral health personnel, and health care providers; and distributing informational pocket cards to homeless persons that described the dangers of using SCs. Anecdotal reports from health care providers 


\section{Summary}

What is already known about this topic?

Synthetic cannabinoids (SCs) are a chemically diverse class of designer drugs that bind to the same cannabinoid receptors as tetrahydrocannabinol (THC), the main psychoactive component of cannabis; some of these drugs are more potent and efficacious agonists than THC and have been associated with outbreaks of overdose-related illness in multiple states. The most commonly reported mode of use involves smoking herbaceous materials that have been sprayed with SCs in liquid form.

What is added by this report?

This investigation demonstrates that responding to outbreaks of adverse SC reactions can place considerable strain on emergency health care systems in affected localities, and highlights the potential for widespread use and associated illness among homeless persons who might be predisposed to adverse health outcomes. In addition, the simultaneous circulation of multiple SC chemicals during an outbreak might influence the variations and severity of illnesses among users. Collection of SCs in liquid form, and anecdotal reports of inhalation of such liquids using a refillable electronic cigarette, provide evidence of a mode of use distinct from smoking of SCs in herbal form.

What are the implications for public health practice?

Pre-established working relationships among public health practitioners, social service providers, EMS personnel, hospital emergency department personnel, law enforcement officials, and laboratory scientists will expedite establishment of the multiagency collaborations needed to respond quickly and comprehensively to outbreaks. Rapidly identifying and focusing prevention programs on vulnerable user groups (e.g., homeless populations) can improve the efficacy and public health outcomes of outbreak interventions.

indicated that information conveyed through educational outreach improved awareness and facilitated diagnosis of incident cases. Educational outreach campaigns focused on the considerable health risks of SC use need to complement judicial and law enforcement actions to reduce SC use, particularly among groups at high risk, such as homeless populations.

\section{Acknowledgments}

Staff members of Anchorage hospitals, Anchorage Department of Health and Human Services, Anchorage Police Department, Alaska State Public Health Laboratory, Alaska State Medical Examiner's Office, and local homeless service organizations; Donna Fearey, Alaska Division of Public Health; Amy Kasper, Tracie Gardner, CDC; Thomas Dobbs, Mississippi State Department of Health.

\footnotetext{
${ }^{1}$ Section of Epidemiology, Alaska Division of Public Health; ${ }^{2}$ Epidemic Intelligence Service, CDC; ${ }^{3}$ Clinical Toxicology and Environmental Biomonitoring Laboratory, University of California, San Francisco; ${ }^{4}$ Anchorage Fire Department, Alaska; ${ }^{5}$ Alaska Native Epidemiology Center, Anchorage, Alaska; ${ }^{6}$ Department of Radiation Oncology, Stanford University School of Medicine, California; ${ }^{7}$ Alaska Department of Health and Social Services.
}

Corresponding author: Louisa J. Castrodale, louisa.castrodale@alaska.gov, 907-269-8000.

\section{References}

1. Law R, Schier J, Martin C, Chang A, Wolkin A. Notes from the field: increase in reported adverse health effects related to synthetic cannabinoid use-United States, January-May 2015. MMWR Morb Mortal Wkly Rep 2015;64:618-9.

2. CDC. Notes from the field: severe illness associated with reported use of synthetic marijuana - Colorado, August-September 2013. MMWR Morb Mortal Wkly Rep 2013;62:1016-7.

3. CDC. Notes from the field: Severe illness associated with synthetic cannabinoid use-Brunswick, Georgia, 2013. MMWR Morb Mortal Wkly Rep 2013;62:939.

4. CDC. Acute kidney injury associated with synthetic cannabinoid usemultiple states, 2012. MMWR Morb Mortal Wkly Rep 2013;62:93-8.

5. Kasper AM, Ridpath AD, Arnold JK, et al. Severe illness associated with reported use of synthetic cannabinoids-Mississippi. MMWR Morb Mortal Wkly Rep 2015;2105:1121-2. http://dx.doi.org/10.15585/ mmwr.mm6439a7

6. Castellanos D, Gralnik LM. Synthetic cannabinoids 2015: an update for pediatricians in clinical practice. World J Clin Pediatr 2016;5:16-24. http://dx.doi.org/10.5409/wjcp.v5.i1.16

7. Longworth M, Banister SD, Mack JBC, Glass M, Connor M, Kassiou $\mathrm{M}$. The 2-alkyl-2H-indazole regioisomers of synthetic cannabinoids AB-CHMINACA, AB-FUBINACA, AB-PINACA, and 5F-AB-PINACA are possible manufacturing impurities with cannabimimetic activities. Forensic Toxicol 2016;34:286-303. http://dx.doi.org/10.1007/ s11419-016-0316-y

8. Adamowicz P, Gieron J. Acute intoxication of four individuals following use of the synthetic cannabinoid MAB-CHMINACA. Clin Toxicol (Phila) 2016;54:650-4. http://dx.doi.org/10.1080/15563650.2016.1190016

9. Banister SD, Moir M, Stuart J, et al. Pharmacology of indole and indazole synthetic cannabinoid designer drugs AB-FUBINACA, ADBFUBINACA, AB-PINACA, ADB-PINACA, 5F-AB-PINACA, 5F-ADBPINACA, ADBICA, and 5F-ADBICA. ACS Chem Neurosci 2015;6:1546-59. http://dx.doi.org/10.1021/acschemneuro.5b00112 